\title{
Althusser con Spinoza: el estatuto material del inconsciente
}

\section{Althusser with Spinoza: the material statute of the unconscious}

\author{
Por: Brodsky, Valentín* \\ Universidad Nacional de Córdoba \\ CONICET \\ Córdoba, Argentina \\ Email: brodskyvalentin@gmail.com
}

Fecha de recepción: 6/04/2019

Fecha de aprobación: 12/05/2020

DOI: $\underline{10.30972 / n v t .1614350}$

\section{Resumen}

El objetivo de este artículo consiste en desplegar algunos elementos que se desprenden del encuentro entre Althusser y Spinoza, para repensar al psicoanálisis en clave estructuralista y materialista. En primer lugar, reconstruiré la forma en la que Althusser vincula al marxismo y al psicoanálisis, en un escrito de 1976 titulado Sobre Marx y Freud. Este texto es sumamente interesante, puesto que el concepto de lo "transindividual" es introducido como el punto de articulación entre los descubrimientos del autor de La interpretación de los sueños y los descubrimientos del autor de El Capital. En un segundo momento de mi trabajo, recuperaré algunos elementos presentes en Tres Notas sobre la teoría de los discursos, para ampliar el concepto de transindividualidad, en el marco de lo que podría denominarse como un "estructuralismo spinozista".

Palabras clave: Spinoza, Althusser, Inconsciente, Estructuralismo, Materialismo, Transindividualidad.

\footnotetext{
* Licenciado en Filosofía por la Universidad Nacional de Córdoba y estudiante del Doctorado en Filosofía de la misma casa de estudios. Forma parte del grupo de investigación "Spinozismo contemporáneo: ontología, realismo y democracia", radicado en el Centro de Investigaciones "Maria Saleme de Burnichon" (CIFFyH-FFyH-UNC). Es adscripto en la catedra de Psicoanálisis y ayudante alumno en Psicología Evolutiva de la Niñez, en la Facultad de Psicología de la UNC. Becario doctoral del Consejo Nacional de Investigaciones Científicas y Técnicas (CONICET, Argentina).
} 


\section{Abstract}

The objective of this article is to display some elements that emerge from the encounter between Althusser and Spinoza, to rethink psychoanalysis in a structuralist and materialist key. First, I will reconstruct the way in which Althusser links marxism and psychoanalysis, in a 1976 writing entitled On Marx and Freud. This text is extremely interesting, since the concept of the "transindividual" is introduced as the point of articulation between the discoveries of the author of The Interpretation of Dreams and the discoveries of the author of Capital. In a second moment of my work, I will recover some elements present in Three Notes on the theory of discourses, to expand the concept of transindividuality, within the framework of what could be called a "spinozist structuralism".

Keywords: Spinoza, Althusser, Unconscious, Structuralism, Materialism, Transindividuality.

\section{Cómo citar este artículo:}

APA: Brodsky, V. (2020). Althusser con Spinoza: el estatuto material del inconsciente, 16 (1), 176-197. Recuperado de: (agregar dirección web)

\section{Delimitación del problema}

El estructuralismo no es una escuela, un movimiento homogéneo, ni posee unx fundadorx ${ }^{1}$. Las diversas obras que han sido agrupadas y reagrupadas bajo ese nombre, al margen de los préstamos conceptuales y afinidades que puedan detectarse entre ellas, poseen una riqueza que desborda los límites de semejante categorización. En este sentido, Balibar (2005) define al estructuralismo como una aventura que dejó

\footnotetext{
${ }^{1}$ En este trabajo trataré de hacer un uso inclusivo y no discriminatorio del lenguaje. Esta decisión política tiene su fundamento en una serie sumamente rica de discusiones que se gestaron recientemente en la Universidad Nacional de Córdoba. Algunos de los efectos de ese debate pueden constatarse en una resolución del Consejo Superior fechada en Septiembre de 2019 (http://www.digesto.unc.edu.ar/consejo-superior/honorable-consejosuperior/resolucion/1095 2019/at download/file). También puede consultarse el documento institucional elaborado por la "Comisión uso inclusivo de la lengua" (https://ffyh.unc.edu.ar/alfilo/consideraciones-sobre-el-uso-inclusivo-de-la-lengua/).
} 
su marca en la historia del pensamiento filosófico, un encuentro entre problemáticas, donde las figuras de Lacan y de Althusser ocupan un lugar de central importancia.

En efecto, como han afirmado otros investigadores (Gillot, 2010), Althusser fue crucial para el pensamiento estructuralista francés de los años '60 y '70, debido a su reactualización del marxismo desde una perspectiva anti-humanista, pero también gracias a su recuperación del psicoanálisis, desde el campo de la filosofía materialista y en clave lacaniana.

Si nos remitimos a un texto de 1963 titulado Filosofía y Ciencias Humanas, encontraremos una nota al pie de página donde Althusser nos indica lo siguiente:

Marx fundó su teoría sobre el rechazo del mito del «Homo oeconomicus». Freud fundó su teoría sobre el rechazo del mito del «Homo psychologicus». Lacan vio y comprendió la ruptura liberadora de Freud. La comprendió en el sentido pleno de la expresión, tomando la palabra en su rigor, y forzándola a producir sin tregua ni concesión, sus propias consecuencias. Él puede, como cualquiera, errar en el detalle, incluso en la elección de las referencias filosóficas: le debemos lo esencial (Althusser, 1998).

Estamos ante un elogio de Freud y Lacan, que atraviesa buena parte de la obra de Althusser. En una primera aproximación, lo que ese elogio pone en juego podría ser explicado como el establecimiento de un paralelismo (Morfino, 2019) o una analogía (Guillot, 2010) que funciona en dos niveles complementarios. En un primer nivel, diremos que la operación que realiza Marx para fundar el objeto de su teoría por fuera del mito del Homo oeconomicus, se produce de manera paralela a la operación que realiza Freud para fundar el objeto del psicoanálisis por fuera del mito del Homo psycologicus. En un segundo nivel, diremos que el retorno a Marx que efectúa Althusser, se despliega en paralelo con el retorno a Freud que realiza Lacan. Hasta aquí el asunto parece bastante simple: Marx y Freud son los fundadores de dos teorías científicas, mientras que Althusser y Lacan son los encargados de garantizar la rectificación y la correcta comprensión de las mismas.

Sin embargo, esta explicación por medio del recurso al paralelismo puede resultar engañosa. La relación entre estos cuatro autores no es tan simple como parece. El vínculo que Althusser establece con el psicoanálisis se encuentra marcado 


\section{Valentín Brodsky}

por cierta "ambivalencia" (Gillot, 2010)‥ Por un lado, Althusser toma diferentes conceptos del campo del psicoanálisis para pensar al marxismo por fuera de la sombra de Hegel. Por otro lado, podemos constatar que este intercambio conceptual se desarrolla de manera paralela con el objetivo althusseriano de fundar epistemológicamente a las ciencias humanas y al psicoanálisis (Balibar, 2005).

Teniendo en cuenta la complejidad del asunto y tomando los recaudos metodológicos apropiados, en las siguientes paginas ensayaré dos grandes movimientos teóricos. En primer lugar, reconstruiré la forma en la que Althusser vincula al marxismo y al psicoanálisis, en un escrito de 1976 titulado Sobre Marx y Freud (1993d). Este texto es sumamente interesante, puesto que el concepto de lo "transindividual" es introducido como el punto de articulación entre los descubrimientos del autor de La interpretación de los sueños (1991) y los descubrimientos del autor de El Capital (2000). En un segundo momento de mi trabajo, recuperaré algunos elementos presentes en Tres Notas sobre la teoría de los discursos (Althusser, 1993e), para ampliar el concepto de transindividualidad, en el marco de lo que podría denominarse como un "estructuralismo spinozista" (Fourtounis, 2005; Steimberg, 2017).

\section{Primera Parte}

Desde el 1 al 5 de octubre de 1979, la Universidad de Tiflis, la Academia de Ciencias de Georgia y el Centro de Medicina Psicosomática Déjerine de París, organizaron un Simposio Internacional sobre el Inconsciente. Gracias al trabajo histórico de Roudinesco (1993), sabemos que Lacan y Althusser fueron invitados a este evento en la ciudad de Tiflis, aunque ambos declinaron la propuesta. En el caso específico de Althusser, lo que motivó su rechazo fue una serie de desafortunados desencuentros editoriales con León Chertok, que dieron lugar a lo que se conoce como

\footnotetext{
${ }^{2}$ A esta relación ambivalente, se le suma el problema de que Althusser modifica su propia posición, a lo largo del tiempo. En términos metodológicos, la tarea de analizar los avatares de la relación entre el marxismo y el psicoanálisis, dentro del conjunto del corpus althusseriano, excede los límites de este artículo
} 
Althusser con Spinoza: el estatuto material del inconsciente

el "affaire Tiflis" y dejaron como saldo dos textos sumamente diferentes: El descubrimiento del doctor Freud (1993b) y Sobre Marx y Freud (1993d).

Si centramos nuestra atención en este último escrito, observaremos que Althusser intenta establecer algunos puntos de contacto entre el autor de El Capital y el autor de La interpretación de los sueños, para superar las resistencias sintomáticas de su época. Al calor de la coyuntura, este filósofo francés ensaya un recorrido de lectura sumamente interesante, por los senderos paralelos que abren Marx y Freud. Ese recorrido puede ser dividido en tres grandes partes:

A)

El primer punto de contacto entre Marx y Freud, en contra de lo que podría creerse, consiste en su falta de originalidad. No hay "originalidad" porque la idea misma de "origen" pertenece a un campo semántico-teórico que resulta incompatible con una concepción no genética, ni subjetiva del conocimiento ${ }^{3}$. Veamos la siguiente cita:

ni Marx ni Freud inventaron nada: el objeto cuya teoría elaboraron respectivamente existía antes de su descubrimiento ¿Qué aportaron?: la definición de su objeto, de su limitación y su extensión, la caracterización de sus condiciones, de sus formas de existencia y de sus efectos, la formulación de las condiciones para comprenderlo y para actuar sobre él: en resumen, su teoría, o las primeras formas de esta (Althusser, 1993d).

El gran descubrimiento de Marx es el materialismo histórico. Su obra contiene, entre otras cosas, una teoría científica sobre las formas, efectos y condiciones de la lucha de clases. Pero, ¿es realmente Marx el 'origen' de esa teoría? ¿No hay algo del orden de lo ideológico y lo teológico en esta singular forma de hablar sobre la 'creación' de una teoría? Apoyándose en Spinoza, Althusser (1993d) afirma que la lucha de clases y sus efectos ya habían sido analizados por otros filósofos políticos, como es el caso de Maquiavelo. También se nos indica que El Capital reposa sobre una lectura crítica de la economía clásica y de los historiadores burgueses de la restauración. Entonces, ¿̇en qué medida podemos establecer una relación de 'filiación'

\footnotetext{
${ }^{3}$ Un desarrollo más acabado sobre el problema que representa el término "origen" puede encontrarse en una carta del 22 de agosto de 1966 que Althusser dirige a Rene Diatkine (1993c).
} 
entre Marx, sus obras y la definición de los objetos científicos que ellas alojan? O, para decirlo en términos spinozistas que recuerdan al Axioma 2 de la Segunda Parte de la Ética (Spinoza, 2011): ¿qué decimos cuando decimos que los seres humanos piensan?

El caso de Freud es analizado de la misma manera por Althusser (1993d). El gran descubrimiento freudiano es el inconsciente. Los escritos, cartas y manuscritos de este autor vienés contienen una teoría científica sobre el inconsciente, sus efectos y las leyes que rigen a esas formaciones. Pero aquí tampoco podemos pasar por alto el hecho de que los efectos del inconsciente ya habían sido reconocidos desde la antigüedad, a través del arte de interpretar los sueños, de la adivinación y de las posesiones. Desde las primeras etapas de su obra, de manera sugerente, Freud (1992) no puede más que reconocer que las palabras provenientes de la relación entre Ixs profesionales de la salud y Ixs pacientes, extrañamente funcionan como un "ensalmo" o un "hechizo" (Zauberei), capaz de contribuir en la curación.

Volvemos a preguntar: ¿qué relación mantienen Marx y Freud con los objetos científicos que han estructurado? Si nos guiamos por lo que afirman algunxs especialistas (Gainza, 2014; Karsz, 1970), observaremos que esta pregunta puede resolverse recurriendo a la distinción entre prácticas, ideología y ciencia, que Althusser introduce en La Revolución teórica de Marx (1967) y en Ideología y Aparatos ideológicos del Estado (2008). Avancemos lentamente: a un nivel general, Althusser (1967) define a las prácticas como procesos históricos de transformación, que poseen un carácter no subjetivo y se ejercen sobre ciertas materias primas. La transformación de esas materias primas, depende de la especificidad de una forma histórica de trabajo humano y de una serie de medios de producción determinados.

La ideología es la forma primaria en que se nos da el mundo. Se trata del modo en que representamos lo que hay, a partir de las transformaciones (y deformaciones) que toda sociedad ejerce sobre la relación imaginaria que enlaza a los seres humanos con la realidad. En este sentido, Althusser (2008) considera que la ideología es la articulación material e histórica de aparatos, instituciones y prácticas, que produce nuestra experiencia inmediatamente vivida. La forma en la que individuos, grupos y 
clases, inconscientemente se representan su relación imaginaria consigo mismxs y con los demás seres que pueblan el mundo, es lo que les permite soportar, justificar o mantener el orden social establecido (Karsz, 1970).

No es posible eliminar por completo a las elaboraciones ideológicas de la sociedad. No hay un grado cero de lo ideológico. A diferencia de lo que han afirmado algunxs representantes del marxismo clásico, para la teoría althusseriana (2008), "ideología" no es un sinónimo de "falsa consciencia", ni debe comprenderse a partir de la metáfora arquitectónica que separa entre infraestructura y superestructura. Aquí no hay una concepción mecánica y economicista para explicar la causalidad que rige a los fenómenos sociales. Por el contrario, la ideología está ligada a nuestra experiencia inmediata y deseante del mundo, a la dimensión material de ciertos aparatos, instituciones, prácticas y rituales que la producen.

Por su parte, lo distintivo de la ciencia es la relación radical y transformadora que mantiene con lo ideológico (Althusser, 1967; Karsz, 1970). La ciencia no parte del objeto real-empírico. Su inicio no se encuentra en los simples hechos, en los datos puros de la conciencia, ni en la percepción directa y sin mediaciones de los objetos (si es que hay algo como eso). La práctica científica es un proceso, basado en una combinación específica de trabajo humano y medios de producción, cuyo objetivo es transformar las representaciones ideológicas de una sociedad histórica determinada, para producir su objeto-de-conocimiento y su efecto-de-conocimiento. El conocimiento científico es el resultado de la aplicación de un método, de la formulación de problemas (en el marco de una problemática determinada) y de la utilización de una serie de instrumentos para resolverlos (como la crítica de la ideología, la abstracción, la activación de una estructura conceptual, etc.).

Ahora bien, después de este breve recorrido, al retornar a la intervención que Althusser (1993d) prepara para el Simposio en Tiflis, podemos entender mejor el primer punto de contacto que establece entre Marx y Freud: estos autores no inventaron nada. En tanto que individuos, forman parte de un amplio proceso histórico-social de producción de conocimiento, que no posee un carácter subjetivo ni 
genético. Si la sociedad es una totalidad compleja, articulada en múltiples niveles y ritmos temporales relativamente autónomos, lo que se condensa bajo los nombres Marx y Freud (al margen de toda distorsión ideológica en torno al problema de la subjetividad) es una modificación estructural en el entramado de las prácticas: "con Marx y Freud, las teorías científicas súbitamente comienzan a ocupar las regiones hasta entonces reservadas a las formaciones teóricas de la ideología burguesa (economía política, sociología, psicología)”. (Althusser, 1993d, p. 222).

B)

El segundo punto de contacto que Althusser establece en Sobre Marx y Freud nos sitúa ante la cuestión del materialismo y la dialéctica:

Todavía vivimos -en todo caso somos muchos los que vivimos- con el mismo presentimiento: demasiadas cosas los aproximan, debe haber algo en común entre Marx y Freud. Pero, ¿qué? [...] Podemos afirmar en primera instancia que, en un mundo igualmente dominado por el idealismo y el mecanicismo, Freud nos ofrece, al igual que Marx, el ejemplo de un pensamiento materialista y dialéctico. (Althusser, 1993d, pp. 224)

Freud como ejemplo del pensamiento materialista y dialéctico. Esta idea de Althusser puede resultar desconcertante. De hecho, uno de los párrafos que siguen a este pasaje y que extraen sus conclusiones, fue censurado durante el "affaire Tiflis" ¿Qué es lo que está afirmando Althusser? Nuevamente, hay que avanzar despacio. Freud es un representante del materialismo, por diversas razones (Althusser, 1993d, p. 224): porque afirma que la realidad existe con independencia del pensamiento humano; porque considera que el proceso de conocimiento no se encuentra regido por la conciencia; y porque, a contra marcha de las elucubraciones ideológicas que rigen en el campo de la psicología, nos proporciona una teoría científica sobre el aparato psíquico, donde el yo (moi) es una de sus partes o efectos, pero no posee un lugar privilegiado.

En resumidas cuentas, el procedimiento de Althusser consiste en recuperar algunas ideas que ya había presentado en otros momentos de su obra, para introducirlas en un nuevo marco de análisis. Si nos remitimos a la primera intervención que realiza durante el año académico de 1963-64, en su Seminario sobre Lacan y el 
Psicoanálisis (1996), observaremos ciertas afinidades: el primer punto que me interesa remarcar, es el hecho de que el problema típicamente estructuralista de la separación entre los campos disciplinares (Balibar, 2005), vuelve a emerger con total claridad. Uno de los objetivos explícitos de Althusser consiste en analizar la separación de hecho y de derecho, entre el psicoanálisis y las ciencias humanas. El segundo punto que considero relevante (Althusser, 1996), es que la figura de Freud vuelve a enlazarse con el descubrimiento del inconsciente y con la posibilidad de una fundación científica del psicoanálisis y la psicología. Pero, quizá lo más importante es que Althusser (1996) reivindica el retorno a Freud efectuado por Lacan, como la única vía posible para preservar el sentido del descubrimiento freudiano y profundizar en la producción de conocimiento científico.

Recordemos que el 15 de enero de 1964, en su apertura del Seminario 11 en L'École des hautes études, Lacan (2010) se detiene exclusivamente a reflexionar sobre su "excomunión" de la IPA y sobre el herem de Spinoza. Como afirman algunas historiadoras (Roudinesco, 2000), Althusser no sólo le facilitó el acceso a ese espacio académico, sino que incluso elogió el resultado de sus investigaciones durante su propio seminario, con el propósito de consolidar una alianza entre ambos. En efecto, siguiendo a Hernández Delgado (2019), creo que es posible afirmar que el retorno y la rectificación de los textos de Marx que encontramos en la filosofía althusseriana, tiene ciertas similitudes con el gesto emprendido por Lacan: el "corte" de Freud, la "ruptura" efectuada con las filosofías de la conciencia y el mito del Homo psychologicus, no es algo permanente. La cientificidad del psicoanálisis no puede fundarse de manera definitiva. La biología, la neurología, las ciencias humanas y la filosofía dominantes en Francia, efectúan diferentes intentos ideológicos para fagocitar el objeto de esta nueva región. En ese contexto, Lacan vuelve a producir una ruptura, colocando al psicoanálisis sobre sus propios pies, sobre una base materialista: afirma que el proceso de conocimiento no es exclusivamente consciente; desmonta el mito del Homo psychologicus, demostrando que la consciencia no es necesariamente el elemento distintivo de la humanidad; y evita reducir al psicoanálisis a una simple técnica de 
adaptación social (camino seguido por Ixs partidarixs de la Ego psychology), mediante una concepción innovadora sobre el Yo.

¿Qué ocurre con la cuestión de la dialéctica? Como lo han demostrado otrxs investigadores (Gillot, 2010; Gainza, 2014), Althusser toma prestados ciertos elementos del psicoanálisis, para elaborar sus propios conceptos de "lectura sintomática", "sobredeterminación" y "causalidad metonímica". Al nivel de su práctica como analista, Freud despliega una forma de pensamiento dialéctico que posee una gran utilidad para pensar al marxismo por fuera de la sombra de Hegel (Althusser, 1993c). Un buen ejemplo de esto podríamos encontrarlo en las famosas páginas que este autor vienés le dedica a esclarecer el "trabajo del sueño" (1991), en su análisis sobre las formas de estratificación del material psíquico y la múltiple determinación de los síntomas en la histeria (1992), pero también en sus reflexiones sobre el caso Dora (1992).

Si nos detenemos brevemente en el sueño de la inyección a Irma, observaremos lo siguiente: Freud trata a esta formación del inconsciente como una totalidad compleja, donde el material onírico se articula mediante una infinidad de conexiones. Cada elemento del contenido del sueño es multívoco, es el subrogado de otros componentes, se encuentra sobredeterminado. En este sentido, constatamos que las figuras de "Irma" y del "Doctor M.", pero también la palabra "propileno", gracias al mecanismo de condensación, toman consistencia después de pasar por un difícil proceso de trabajo. "Irma" es una imagen de acumulación [Sammelbild], puesto aloja y soporta un conjunto de rasgos pertenecientes a diferentes individuos (síntomas, gestos, posiciones corporales, etc.), incluso cuando ellos se contradicen entre sí. Por su parte, el término "propileno" nos permite entender el modo en que las palabras arman circuitos y conforman puntos nodales, estableciendo las conexiones que sostienen a la trama del sueño, y que luego caerán en el olvido con el retorno a la vigilia.

Lo que Althusser encuentra en esta clase de investigaciones freudianas es, entre otras cosas, una valiosa herramienta para pensar científicamente a la historia y 
desprenderse de las estrategias mediante las que tradicionalmente la filosofía había explicado a la relación entre la estructura y sus efectos. Contra el modelo de la causalidad transitiva (de origen galileano), pero también contra el modelo de la causalidad expresiva (proveniente de Leibniz y Hegel), Marx nos ofrece un análisis innovador: las formaciones históricas son totalidades complejas, regidas por una estructura a dominante. El mundo se despliega bajo múltiples niveles, y cada uno de ellos se rige según una temporalidad propia: esas diferentes dimensiones poseen una autonomía relativa y una historia diferencial, donde el ritmo y las leyes son determinadas por las conexiones (de correspondencia, no correspondencia, articulación, desplazamiento, torsión, etc.) que se den hacia el interior del todo social.

Dentro de esa misma línea de intercambios y préstamos teóricos entre Marx y Freud, me parece relevante destacar el hecho de que Lacan (2003) se anticipa algunos años a Althusser cuando define al psicoanálisis como "una experiencia dialéctica". Creo que Intervención sobre la transferencia es sumamente interesante para observar la forma en que el psicoanálisis lacaninano reactualiza la ruptura de Freud: el texto presenta una crítica a las concepciones ideológicas sobre el manejo de la transferencia que postula el psicoanálisis norteamericano, al mismo tiempo que asienta algunos elementos conceptuales, extraídos y elaborados a partir de un trabajo sumamente cuidadoso en el terreno de la clínica. En este sentido, la articulación entre los desarrollos de verdad y las sucesivas inversiones dialécticas presentes en el caso Dora, son el efecto de las investigaciones científicas que Lacan realiza para retornar a Freud y rectificar la práctica psicoanalítica.

C)

El tercer punto de contacto mencionado por Althusser en Sobre Marx y Freud, consiste en el hecho de que estamos ante dos ciencias conflictivas y divisorias:

¿Esas afinidades teóricas son suficientes para dar cuenta de la comunidad teórica que existe entre Marx y Freud? Si y no. En efecto, podríamos detenernos ahí (el balance filosófico ya es rico), y dejar que cada teoría funcione por su lado, es decir que afronte su propio objeto, irreductible en tanto que objeto a las afinidades filosóficas de las que acabamos de hablar, y retirarnos para callar. Sin embargo, otro fenómeno aún más sorprendente debe retener nuestra atención: es lo que 
llamé el carácter conflictivo de la teoría marxista y de la teoría freudiana.

(Althusser, 1993d, p. 225)

La conflictividad es inherente a la teoría marxista, porque esta última surge de la división de clases que atraviesa a la sociedad. A distancia de aquella concepción ideológica que presenta a la práctica científica como basada en una posición neutral, Marx entiende que la esencia de la realidad es conflictual y que ella solo puede estudiarse a partir de una toma de posición en el entramado social (incluso cuando esa posición no es consciente o explícitamente asumida). El ejercicio marxista de dividir, es decir de intervenir en el conflicto y producir separaciones mediante la práctica teórica, es algo que debe reactivarse cada vez, nuevamente, en cada coyuntura.

Por su parte, si continuamos con el desarrollo de la lectura althusseriana, diremos que el psicoanálisis es una ciencia conflictiva porque toca el núcleo sensible de la ideología filosófica, psicológica y económica, que justifica el orden de desigualdades sobre el que se construye la sociedad. El descubrimiento del inconsciente y sus efectos, atenta contra la idea de lo humano, contra la idea de un sujeto unitario que se distingue del resto de los seres que pueblan el mundo debido a la conciencia que tiene de sí. En palabras de Althusser:

En verdad, si uno comprende bien a Marx, no hay ningún misterio en ese "punto sensible" que Freud dañó en toda la tradición de la filosofía clásica y en las formaciones teóricas de la ideología burguesa, como la psicología, la sociología y la economía política, o en sus formaciones prácticas como la moral y la religión. Basta con comprender que los diferentes "sujetos-conscientes-de" son unificadores de la identidad social del individuo en tanto que ellos son unificados como ejemplares de una ideología del "hombre". (Althusser, 1993d, p. 235-236)

La "tesis de oro" del materialismo consiste colocar el acento en la realidad, no en la conciencia (Althusser, 1993d). El materialismo nos conduce a juzgar a los entes por el conjunto de condiciones materiales que los producen, y no por la conciencia que tienen de sí (ya que esta última es siempre una función de desconocimiento). En tanto que la ideología atraviesa las diferentes regiones que constituyen a la totalidad social, la consciencia aparece como el elemento que corona a la idea de lo humano. Althusser reconoce diferentes unificadores de la identidad social del individuo: la idea del Homo economicus (como sujeto-consciente-de-sus-deseos), la idea del sujeto moral 
(entendido como sujeto-consciente-de-sus-actos), pero también la idea del sujeto de derechos (es decir, sujeto que posee una identidad, es consciente de las leyes que lo constriñen y puede dar cuenta de ellas). "Consciente de si" es el nombre de una función delegada al individuo, para articular y justificar las diversas prácticas cognitivas, políticas y morales de una sociedad histórica, que produce y reproduce sus desigualdades en una infinidad de niveles.

Se comprenden entonces las enormes resistencias que despierta la obra de Freud, así como también los diferentes intentos que se realizan para apropiarse y neutralizar sus descubrimientos, al trasladarlos hacia el interior de otros campos disciplinares (por ejemplo: la biología o las neurociencias). Para Althusser, si la noción de lo inconsciente resulta inaceptable, esto se fundamenta en dos razones que se articulan a diferentes niveles del entramado social: en primer lugar, diremos que el objeto del psicoanálisis resulta inaceptable y es reprimido, puesto que la consciencia funciona como una exigencia política e ideológica, destinada a mantener un sistema de desigualdades. Pero, en segundo lugar, debemos reconocer que el inconsciente también es reprimido por el aparato psíquico propio de cada individuo. Puede que el masivo rechazo del psicoanálisis, por parte de filósofxs marxistas, médicxs, psicólogxs y moralistxs, no se deba solo a un antagonismo ideológico de masa, sino que también se encuentre implicado el hecho de que cada uno de estos individuos posee un inconsciente que desconoce, nos dice Althusser, mediante una cierta compulsión a la repetición, la misma idea de que el inconsciente existe.

\section{Segunda Parte}

En función de este recorrido que realiza la lectura althusseriana, vemos que los análisis de Marx y Freud se aproximan, sin llegar confundirse ni homologarse. Son como dos rectas paralelas: no se tocan, pero siempre podemos dibujar nuevas líneas que unan algunos puntos ¿Qué ocurre con el problema de las separaciones disciplinares? Mi hipótesis, como dije en la introducción, consiste en afirmar que ese 
vínculo entre marxismo y psicoanálisis se completa en la obra de Althusser mediante la recuperación de ciertos elementos de la obra de Spinoza.

Para justificar esta última afirmación de la manera más clara posible, trataré de apegarme al texto:

La paradoja es que Freud, salvo algunos ensayos dudosos y discutibles (Totem y tabu, El malestar en la cultura, etc.), nunca trató verdaderamente de abarcar y pensar como un todo a esta ideología burguesa a la que hería en su punto más sensible. Vayamos más lejos: no estaba en condiciones de hacerlo, porque para eso hacía falta que fuera Marx. No era Marx: tenía un objeto totalmente diferente. Pero le bastó con revelar al mundo estupefacto que este otro objeto existía, para que las consecuencias se sacaran por sí mismas. (Althusser, 1993d, p. 236)

Y un poco más adelante, deslizando rápidamente el concepto de transindividualidad, Althusser sostiene lo siguiente:

en estos ensayos de generalización desafortunada, Freud no dejó de repetir en condiciones discutibles lo que había descubierto en otra parte. Lo que había descubierto no trataba en absoluto de la "sociedad" o de las "relaciones sociales", sino más bien de los fenómenos sumamente particulares que afectaban a los individuos. Aunque se hubiera podido sostener que en el inconsciente hay un elemento "transindividual", de todas formas, es en el individuo donde se manifiestan los efectos del inconsciente, y es sobre el individuo que opera la cura [...] Esa diferencia basta para distinguir a Freud de Marx. (Althusser, 1993d, p. 238)

Estas citas contienen al menos dos elementos de gran importancia: en primer lugar, Althusser sostiene que el error cometido por Freud en algunos de sus ensayos (por ejemplo: Tótem y Tabú, El malestar en la cultura, etc.), consiste en tomar ciertos fenómenos pertenecientes al terreno de la clínica, para desplazarlos y generalizarlos a nivel social. Marx y Freud fundan dos teorías, pertenecientes a regiones científicas diferentes. El psicoanálisis (y lo mismo vale para el marxismo) es sólo una ciencia regional, no el fundamento teórico de todas las humanidades y ciencias sociales.

El segundo punto importante que se desprende de estos pasajes, es el nexo que Althusser establece entre inconsciente y transindividualidad. Nuevamente, algunos interrogantes surgen en el camino del análisis. En un primer nivel de lectura, podríamos preguntar: ¿Qué significado tiene el concepto de transindividualidad? ¿Cuáles son las fuentes a las que Althusser recurre para delimitar esta noción? Luego, 
situándonos en otro nivel, quizá también sería razonable preguntar: ¿En qué sentido afirma Althusser que el inconsciente es transindividual? ¿De qué modo podemos sostener que los síntomas o los sueños de un individuo se articulan causalmente con el entramado social al que pertenece, sin confundir los hallazgos de Marx y Freud?

Comienzo por el primer grupo de interrogantes. Althusser no nos ofrece una definición precisa del concepto de transindividualidad en este texto y debo confesar que, hasta el momento, no he sido capaz de localizar otra aparición del término en sus obras. En este sentido, es importante recurrir al trabajo desarrollado por otrxs investigadores (Balibar, 2000; 2009; 2018; Balibar \& Morfino, 2014; Gainza, 2016): dentro de la historia del pensamiento, no existe una tradición de la transindividualidad claramente delimitada. La expresión puede rastrearse en Función y campo de la palabra y del lenguaje en psicoanálisis de Lacan. Pero, si deseamos encontrar un trabajo conceptual de carácter sistemático, quizá lo más apropiado sería dirigir nuestra atención hacia La individuación psíquica y colectiva de Gilbert Simondon (2007).

Este libro de Simondon es un intento de refundar a las ciencias sociales, sobre la base de una crítica radical a la noción metafísica de individuo. Transindividualidad es el nombre de una apuesta que se juega en una serie de registros diferentes, pero estrechamente vinculados entre sí. Por un lado, apoyándonos en Balibar (2009), creo que es posible afirmar que lo transindividual representa la posibilidad rehabilitar el proyecto althusseriano de una fundación ontológico-epistémica para las ciencias sociales, dejando de lado algunas oposiciones duales que han caracterizado al pensamiento contemporáneo (individualismo/holismo, psicologismo/sociologismo, interioridad/exterioridad). Por otro lado, siguiendo a Morfino (2010), creo que lo transindividual nos sitúa ante la posibilidad de pensar una ontología de la relación que, siempre bajo la tutela de la tesis del primado del encuentro sobre la forma, permite un rastreo genealógico que llega hasta Spinoza y Marx (entre otros autores).

Ahora bien, pasemos al segundo grupo de preguntas. Si suspendemos momentáneamente esta esquemática exploración histórico-conceptual sobre lo 
transindividual y nos concentramos en la filosofía Althusser ¿En qué sentido podríamos decir que el inconsciente es transindividual? Para responder a esta cuestión, creo que es necesario recuperar el texto titulado Tes notas sobre una teoría de los discursos. Este escrito se encuentra fechado alrededor de 1966, pero fue publicado de manera póstuma. Se trata de una serie de notas exploratorias que Althusser escribe y comparte con algunos discípulos (Alain Badiou, Étienne Balibar, Pierre Macherey, Yves Duroux), que poco tiempo después pasarían a integrar una organización teórica y política denominada Groupe Spinoza. El objetivo del escrito era elaborar una nueva obra de filosofía ("Elementos de Materialismo Dialectico") que funcionaría como su Ética, según lo anunciado en una carta a Balibar (Althusser, 1993e).

Es cierto que el proyecto althusseriano de reescribir colectivamente la Ética de Spinoza no llegó a concretarse. Aun así, esas investigaciones y ensayos grupales dejaron como resultado un conjunto de conjeturas sumamente interesantes para pensar el estatuto del inconsciente. A continuación, trataré de reconstruir dos puntos del texto que considero cruciales para comenzar pensar al psicoanálisis en clave marxista y spinozista:

1) Comencemos por delimitar algunos conceptos fundamentales que se encuentran presentes en el título: ¿Qué entendemos por el término discurso? Como han afirmado algunos investigadores (Morfino, 2019), la definición althusseriana de este concepto es sumamente amplia. "Discurso" no nos remite a un simple articulado de letras, palabras y enunciados, sino más bien a una estructura transindividual que conecta cuerpos, afectos, ideas, aparatos y prácticas ritualizadas.

Althusser (1993e) distingue entre el discurso de la ciencia, el discurso estético, el discurso ideológico y el discurso del inconsciente. En un intento de formalización, sostiene que cada uno de esos discursos posee su propia estructura, utiliza cierto tipo de significantes y se define por una función específica ${ }^{4}$. Si tomamos el caso del discurso

\footnotetext{
${ }^{4}$ Es cierto que, en el contexto de la primera nota, Althusser afirma que cada discurso produce su propio efecto-sujeto. Aun así, esta hipótesis es explícitamente rechazada en la carta del 28 de octubre de 1966 que acompañaba la recopilación y el envío de las tres notas (Althusser, 1993e). En el fondo, lo que tenemos aquí es una discusión con Lacan sobre el problema de la división subjetiva. Por razones metodológicas, trataré de abordar este problema en otro trabajo.
} 
del inconsciente, podemos decir que su estructura es metonímica, es decir, una estructura de fuga, donde el sujeto está ausente como "lugarteniente" y la materialidad imaginaria de los significantes configura diversas fantasías. Cada una de nuestras fantasías es una escena de posiciones fijas e imaginadas, construida sobre la base de la sintaxis (condensación y desplazamiento) que es propia del discurso del inconsciente.

El resultado de la articulación imaginaria de las fantasías, gracias a la aplicación de una serie específica de reglas, es el efecto-libido: el deseo no es algo que está antes o detrás de las fantasías, bajo la modalidad de una causa oculta. El deseo es el efecto que se desprende de la singular e irrepetible toma de consistencia de la materialidad imaginaria de las fantasías. En principio, se trata de una forma única y alucinada de ser en el mundo ¿Como es posible entonces que habitemos una realidad donde también hay otrxs?

2) En su esfuerzo por pensar las condiciones materiales en que se constituye el inconsciente y en las que opera su estructura a lo largo de la vida humana, Althusser recurre a Spinoza. En el ensayo titulado Freud y Lacan (1993a), dentro del tercer apartado, hay una nota al pie de página que intenta explicar el funcionamiento del Edipo mediante la noción spinoziana de causa sui. Se trata de una sugerencia interesante: la práctica del psicoanálisis se ejerce sobre las formas singulares en las que se estructura el inconsciente de cada individuo, es decir, sobre los efectos materiales producidos por los diversos modos de devenir-humano que se encuentran habilitados en una determinada época histórica.

En una sociedad capitalista y patriarcal como la nuestra, la vida humana supone la puesta en marcha del Edipo, de una maquinaria violenta e insistente, cuyo propósito consiste en modificar y normalizar la forma que todas las "larvas mamíferas" deben asumir para su progresiva composición con el entramado social ${ }^{5}$. En un sentido

\footnotetext{
${ }^{5}$ Entiendo que el aparato-familia ha experimentado importantes transformaciones con el paso del tiempo y que hoy en día existen múltiples configuraciones familiares. También es cierto que la familia no es el único mecanismo por medio del cual puede producirse el proceso de socialización. Es decir, es posible que otras comunidades encuentren vías diferentes para logar la composición entre un individuo y el entramado socio-histórico que lo aloja. Sería interesante actualizar el análisis althusseriano,
} 
profundamente spinozista, Althusser marca dos características esenciales de la estructuración edípica: en primer lugar, diremos que la estructura no opera como una esencia anterior y/o exterior a las formaciones del inconsciente, sino que se rige por la lógica de la inmanencia. La estructura es causa de sí misma, en el mismo sentido en que es causa de todas las cosas. No tiene existencia por fuera de sus efectos. El Edipo, en tanto que estatuto que rige el inconsciente, sólo adquiere consistencia en el proceso transindividual por medio del cual se constituye cada modo humano, en cada momento de su vida.

En segundo lugar, si retomamos nuestra lectura de Tres Notas..., diremos que la estructura edípica toma consistencia, por primera vez, bajo el conjunto de condiciones materiales específicas en las que cada sujeto realiza su propio pasaje ¿Cuáles son esas condiciones? Veamos lo que escribe Althusser en una carta a Hélène del 30 de julio de 1964:

El tema esencial es evidentemente aquel que concierne a lo que el niño encuentra al nacer, y que habitualmente su nacimiento contribuye a anudar: el nudo del deseo de los padres. $\mathrm{O}$, para dar un sentido todavía más preciso a los términos: el pacto pasado entre los deseos de los padres, el tratado (de guerra) (de paz) pasado entre los deseos de los padres. (Althusser, 2011, p. 447)

Para Althusser, el inicio de la vida del individuo no se encuentra en el nacimiento, sino que más bien reside en el "pacto" entre los deseos de otros individuos que lo anteceden. La existencia de cada niñx se encuentra condicionada por la larga historia vincular que mantiene con sus figuras parentales, dentro de una sociedad histórica determinada. Nacer: verbo que usamos para pensar la llegada al mundo de un individuo, su primer encuentro con lo que hay, en el marco de una totalidad social de relaciones que son preexistentes y lo hacen consistir.

Lo que permite que el individuo haga lazo con el mundo, es la estructuración de su inconsciente hacia el interior de la familia, pero también en relación con otras instituciones. Para Althusser, el atravesamiento del Edipo se produce gracias a la creo que un punto interesante para continuar la conversación puede encontrarse en el diálogo sobre lo transindividual que mantienen Read y Balibar (Read, 2018), pero también en un conocido estudio de Judith Butler (2001) 
articulación entre el discurso del inconsciente y el discurso ideológico. En efecto, en el análisis de su propia época, este autor considera que la institución familiar se encuentra íntimamente conectada a otros aparatos ideológicos del Estado, que también producen efectos materiales sobre el inconsciente. El jardín de infantes y la escuela, son dos ejemplos cruciales dentro de Ideología y Aparatos Ideológicos del Estado. Veamos lo que dice sobre la institución educativa:

Toma a su cargo a los niños de todas las clases sociales desde el jardín de infantes, y desde el jardín de infantes les inculca -con nuevos y viejos métodos, durante muchos años, precisamente aquellos en los que el niño, atrapado entre el aparato de Estado-familia y el aparato de Estado-escuela, es más vulnerable"habilidades" recubiertas por la ideología dominante (el idioma, el cálculo, la historia natural, las ciencias, la literatura) o, más directamente, la ideología dominante en estado puro (moral, instrucción cívica, filosofía). (Althusser, 2003, p. 134)

Para garantizar la reproducción del orden social, junto con la infinidad de diferencias que este esconde o justifica hacia su interior, los aparatos ideológicos producen a los individuos en tanto que sujetos. Desde la perspectiva de Althusser, individuo, sujeto e inconsciente son términos que no deben confundirse, aunque se encuentran íntimamente vinculados. Estamos ante a algo que se parece bastante a un pliegue y el desafío althusseriano consiste precisamente en pensarlo sin recurrir al lenguaje teológico de la génesis, de la filiación: el movimiento por el cual la ideología llama a un individuo para que ocupe un rol en la estructura social, es el mismo movimiento por el cual ese individuo experimenta un efecto-inconsciente y se lo constituye como sujeto.

La estructura social no está interesada en quién ocupa cada uno de los roles que le dan consistencia. El carácter domesticador de la vida cotidiana presupone que ese sujeto-quien existe, fuerza al individuo a ingresar como "portador" (Träger) de sus funciones, y produce un efecto-sujeto que es siempre singular, único e irrepetible. Althusser lo expresa del siguiente modo: "la interpelación como sujetos ideológicos de los individuos humanos produce en ellos un efecto específico, el efecto inconsciente, que permite a los individuos humanos asumir la función de sujetos ideológicos" (1993e, p. 139). 


\section{Conclusión:}

Hasta aquí he tratado de desplegar algunos elementos que se desprenden del encuentro entre Althusser y Spinoza, para repensar al psicoanálisis en clave estructuralista y materialista. En la primera parte del artículo, intenté mostrar las razones por las cuales Althusser lee las investigaciones de Marx y Freud, como dos senderos paralelos, entre los cuales es posible establecer diversos puntos de contacto: el problema de la originalidad, de la dialéctica, del materialismo y del conflicto. En la segunda parte del artículo, intenté recuperar el concepto de transindividualidad, desde lo que podría denominarse como un "estructuralismo spinozista", para pensar el problema del inconsciente.

Este artículo no pretende agotar todo lo que podría decirse sobre la base de la relación Marx-Freud-Althusser-Lacan. Se trata más bien de un trabajo exploratorio y de una invitación al diálogo colectivo. Desde mi perspectiva, creo que sería interesante releer algunos textos de Freud y Lacan, en clave marxista y spinozista, para delimitar teóricamente al menos dos cuestiones. Por un lado, el problema de la estructuración de los afectos humanos y su relación con el inconsciente. Por otro lado, la articulación entre los conceptos de necesidad-demanda-deseo, de manera tal que el psicoanálisis no reproduzca ciertas posiciones antropocéntricas, que es preciso abandonar en la coyuntura actual. 


\section{Referencias Bibliográficas}

Althusser, L. [(2003) 1970]. Ideología y aparatos ideológicos del Estado, Zizek, S. (Comp.), en Ideología: un mapa de la cuestión, Buenos Aires: Fondo de Cultura Económica.

Althusser, L. (2011). Lettres à Hélène 1947-1980. París: Éditions Grasset \& Fasquelle et IMEC.

Althusser, L. [(1996) 1963-64]. Psychanalyse et sciences humaines: Deux conférences (1963-1964), París: Librairie générale française.

Althusser, L. [(1993a) 1964]. Freud et Lacan, en Écrits sur la psychanalyse. Freud et Lacan, París: Editions Stock/IMEC.

Althusser, L. [(1993b) 1976]. La découverte du docteur Freud, en Écrits sur la psychanalyse. Freud et Lacan, París: Editions Stock/IMEC.

Althusser, L. [(1993c) 1966]. Lettre à D... (n²), en Écrits sur la psychanalyse. Freud et Lacan, París: Editions Stock/IMEC.

Althusser, L. [(1993d) 1976]. Sur Marx et Freud, en Écrits sur la psychanalyse. Freud et Lacan, París: Editions Stock/IMEC.

Althusser, L. [(1993e) 1966]. Trois notes sur la théorie des discours, en Écrits sur la psychanalyse. Freud et Lacan, París: Editions Stock/IMEC

Balibar, E. (2000). La Filosofía de Marx, (Pons, H., Trad.) Buenos Aires: Ediciones Nueva Visión.

Balibar, E. (2005). Le structuralisme ¿Une destitution du sujet?, en Revue de métaphysique et de morale, 45, p. 5-22.

Balibar, E. (2018). Philosophies of the Transindividual: Spinoza, Marx, Freud, en Australasian Philosophical Review, 2 (1), p. 5-25.

Balibar, E. (2009). Spinoza: de la individualidad a la transindividualidad, (Torres, A., Trad.) Córdoba: Encuentro Grupo Editor.

Balibar \& Morfino. (2014). Il transindividuale. Soggetti, Relazioni, Mutazioni, Milán: Mimesis.

Butler, J. (2001). Mecanismos psíquicos del poder. Teorías sobre la sujeción, (Cruz, J., Trad.) Madrid: Cátedra.

Fourtounis, G. (2005). On althusser's immanentist structuralism: reading Montag reading althusser reading spinoza, en Rethinking Marxism, 17 (1), p. 101-118.

Freud, S. (1992). Fragmento de análisis de un caso de histeria (1905 [1901]), en Fragmento de análisis de un caso de histeria (Dora). Tres ensayos de teoría 


\section{Valentín Brodsky}

sexual y otras obras (1901-1905), (Etcheverry, J. L., Trad.) Obras Completas, 7, Buenos Aires: Amorrortu editores.

Freud, S. (1992). Estudios sobre la Histeria, (Etcheverry, J. L., Trad.) Obras Completas, 2, Buenos Aires: Amorrortu editores.

Freud, S. (1991). La interpretación de los sueños (Primera parte), (Etcheverry, J. L., Trad.) Obras Completas, 4, Buenos Aires: Amorrortu editores.

Gainza, M. (2016). El límite y la parte: los confines de la interioridad en la filosofía spinoziana, en Demarcaciones, 4, p. 208-219.

Gainza, M. (2014). Zizek y Althusser. Vida o muerte de la lectura sintomática, en Decalages, 1 (1) [Recuperado de: https://scholar.oxy.edu/decalages/vol1/iss1/3].

Gillot, P. (2010). Althusser y el psicoanálisis, (Cardoso, H., Trad.) Buenos Aires: Ediciones Nueva Visión.

Karl, M. (2000). El Capital, (Romano García, R., Trad.) Libro I, Tomo I, Madrid: Akal.

Karsz, S. (1970). "Lectura de Althusser", Karsz, S. (Comp.), en Lectura de Althusser, Buenos Aires: Galerna.

Lacan, J. (2003). Intervención sobre la transferencia, (Segovia, T., Trad.) en Escritos, Ciudad de México: Siglo veintiuno editores.

Lacan, J. (2010). Seminario 11: los cuatro conceptos fundamentales del psicoanálisis, (Delmont-Mauri, J. L. \& Sucre, J., Trad.), Buenos Aires: Paidós.

Morfino, V. (2019). Beyond the 'Repressive Hypothesis': 'Subject' and 'Libido-Effect' in Althusser, (Pipa, S., Trad.) en Rethinking Marxism, № 31, Vol. 3, p. 273-290.

Morfino, V. (2010). Spinoza. Relación y Contingencia, (Torres, S., Trad.) Córdoba: Encuentro Grupo Editor.

Read, J. (2018). The Transindividual Unconscious, en Australasian Philosophical Review, Vol. 2, №1, p. 62-68.

Roudinesco, E. (2010). Lacan. Esbozo de una vida, historia de un sistema de pensamiento, Santafé de Bogotá: Fondo de Cultura Económica.

Roudinesco, E. (1993). La batalla de cien años. Historia del psicoanálisis en Francia, (Guyer, A. E.) Tomo III, Madrid: Editorial Fundamentos.

Simondon, G. (2007). L'individuation psychique et collective, Lonrai: Aubier.

Steimberg, R. (2018). Hacia un estructuralismo Spinozista, en Cuadernos de la Facultad de Humanidades y Cs. Sociales, 53 